\title{
Crystal structure of a bacterial Dicer that is ideal for the preparation of heterogeneous siRNA cocktails
}

\author{
S dharavath ${ }^{1}$ \\ ${ }^{1} N / A, N / A$ \\ dharavaths2@nih.gov
}

Ribonuclease III (RNase III) represents a highly conserved family of double-stranded RNA (dsRNA) specific endoribonucleases that are important for RNA processing and post-transcriptional control of gene expression. The RNase III family includes prokaryotic RNase III and eukaryotic Rnt1p, Drosha, and Dicer, among which the RNase III from Escherichia coli (EcRNase III) is the most comprehensively studied member. It contains a characteristic RNase III domain (RIIID) and a dsRNA-binding domain (dsRBD) and its E38A mutant (EcE38A) has been utilized to produce small interfering RNAs (siRNAs), mimicking the product of Dicer. The siRNA cocktails thus produced, however, were not heterogeneous due to base-specific substrate selection of the enzyme. Further engineering EcE38A, we created a triple mutant of EcRNase III (EcE38A/E65A/Q165A) that does not have sequence specificity and therefore is ideally suited for producing heterogeneous siRNA cocktails to be used in gene silencing studies. Hence, we refer to this triple mutant as a bacterial Dicer. Here, we present the crystal structures of the bacterial Dicer in complex with products of dsRNA cleavage, showing three slightly different cleavage site arrangements and providing structural basis for its Dicer-like properties. There are two independent molecules in the asymmetric unit. The two molecules dimerize to form a catalytic valley that accommodates dsRNA. The RIIID of each subunit cleaves one RNA strand and the cleavage of both strands generates a 2-nucleotide 3' overhang, a typical feature of the product of all RNase III enzymes. EcRNase III was discovered in 1968. The structures reported here represent the first three-dimensional structures of the founding member of the RNase III family.

Acta Cryst. (2020). A76, a48 